\title{
Opinión del profesorado de Lengua Extranjera en torno a la integración del componente cultural en el aula de idiomas
}

\author{
María José CARrillo López \\ Universidad de Málaga
}

Recibido: 20 diciembre 2012 / Aceptado: 15 febrero 2013

ISSN: $1697-7467$

\begin{abstract}
RESUMEN: En este artículo presentamos un estudio que forma parte de una investigación más amplia en la que se indagan las percepciones del profesorado de Lengua Extranjera en torno a la integración del componente cultural en el aula de idiomas. Estas creencias han sido obtenidas mediante un cuestionario que pretendía conocer las opiniones de un colectivo de profesores de las provincias de Málaga y Granada en torno a tres cuestiones fundamentales: la relevancia que la Unión Europea otorga a la dimensión sociocultural; la importancia relativa de los componentes lingüístico y/o cultural en la enseñanza/aprendizaje de una lengua extranjera y el tipo de contenidos que deberían impartirse. De cada ítem se ha realizado un análisis de la distribución de frecuencia y la posible variabilidad de las opiniones que manifiestan los docentes en función del idioma enseñado, de los años de experiencia docente, de la provincia en la que imparten dicha docencia, del nivel de enseñanza y del sexo.

Palabras clave: Enseñanza de la lengua extranjera, creencias, competencia sociocultural, competencia lingüística.
\end{abstract}

Foreign Language Teachers' Beliefs about the Integration of the Cultural Element in the Language Classroom

\begin{abstract}
This paper is part of a wider research in which we study the beliefs of Second Language Teachers about the integration of cultural aspects in the foreign language classroom. A questionnaire were distributed among teachers in the provinces of Malaga and Granada in order to find out what they thought on three fundamental issues: 1) the importance that the European Union gives to the teaching of cultural aspects; 2) the relative weight that linguistic and cultural elements have in Foreign Language Teaching; and 3) which cultural contents should be included in the syllabus. For each item, we perform an analysis of the frequency distribution and the existence of statistically significant differences on their answers according to the independent variables such as language being taught, years of experience, the province in which they teach, the academic level and the gender.

Keywords: Foreign language teaching; teachers' beliefs; sociocultural competence, linguistic competence.
\end{abstract}

\section{INTRODUCCIÓN}

El concepto de 'competencia comunicativa' ha supuesto un referente esencial en la enseñanza de Lenguas Extranjeras (en adelante, LE). No habría sido posible alcanzar la situación actual sin el 'empuje' proveniente de las Ciencias del lenguaje, pues han sido, sin duda, 
estas disciplinas las fuentes principales de las que los metodólogos han obtenido conceptos y teorías que han resultado a la postre cruciales para el avance en el campo de la didáctica (Girard, 1981), y, ¿cómo no? para un mejor conocimiento de lo que implica dominar un idioma. La implantación del enfoque comunicativo (a través de nociones tales como acto comunicativo, intención comunicativa del hablante, contexto situacional, encuentro social, papel del hablante, documento auténtico, vacío de información, actividades interactivas en parejas, juego de rol, etc.) pone todo el énfasis en la comunicación y la funcionalidad del lenguaje. Desde esta perspectiva, para que llegue a producirse el aprendizaje de una lengua extranjera que redunde en su uso eficaz, no basta con acumular conocimientos sobre las 'formas lingüísticas' fuera de un contexto de uso. El enfoque comunicativo aborda, por vez primera, el contexto sociocultural, es decir los marcos de referencia (valores, creencias compartidas, normas sociales, actitudes, etc.) que rigen el uso del lenguaje en una determinada comunidad de habla. El éxito comunicativo en el transcurso de un intercambio depende de una multitud de parámetros que a partir de la formulación de este concepto clave entran en escena (por ejemplo, los marcadores lingüísticos de las relaciones sociales, las convenciones de cortesía, etc.). En esta línea, se pronuncia Saville-Troike (1982: 1), quien específica que para llevar a cabo una comunicación eficiente y apropiada al encuentro social es preciso conocer:

not only rules for communication (both linguistic and sociolinguistic) and shared rules for interaction, but also the cultural rules and knowledge that are the basis for the context and content of communicative events and interaction processes.

En los últimos años un nuevo modelo, el 'enfoque por competencias', orientado hacia la acción y promovido por el Marco común europeo de referencia para las lenguas: aprendizaje, enseñanza, evaluación (en adelante, MCERL) (2002), se abre paso en el campo de la didáctica de las lenguas. Este enfoque metodológico considera la lengua como un proceso dinámico, es decir que invita:

à dépasser un apprentissage de la langue qui serait basée uniquement sur l'intention du locuteur utilisée comme moyen d'action sur l'Autre, sachant qu'une intention ne débouche pas nécessairement sur la réussite d'une action (Bourguignon, 2007) ${ }^{1}$.

El MCERL recomienda prestar la debida atención a la dimensión intercultural teniendo en cuenta que, en nuestras sociedades pluriculturales y multilingües, el usuario de la lengua tendrá que hacer gala de un saber hacer y de un saber ser que le habilite para interactuar eficazmente con sus interlocutores provenientes de una variedad de orígenes y de culturas distintas. En efecto, la diversidad existente tanto en la sociedad como en el propio centro educativo obliga a los y las docentes a tomar conciencia, cada vez más, de ello. El aprendiz de lenguas que habrá de adaptarse a múltiples parámetros situacionales se ha de convertir en un agente social. Por lo tanto, deberá saber comportarse adecuadamente en los distintos

\footnotetext{
${ }^{1}$ Conferencia realizada por Claire Bourguignon, en marzo de 2007 en Grenoble: “Apprendre et enseigner les langues dans la perspective actionnelle: le scénario d'apprentissage-action", disponible en : http://www.aplv-languesmodernes.org/spip.php?article865, consultado en marzo de 2008.
} 
encuentros sociales y culturales en los que se verá envuelto. Así lo expresan Julié y Perrot (2008: 63):

L'héritage et le prolongement le plus significatif qu'apporte la perspective actionnelle à l'approche communicative, $c^{\prime}$ est qu'il faut savoir communiquer certes, mais que cela doit servir un projet plus vaste qui tourne le dialogue entre les individus vers une coopération entre les peuples d'Europe.

Tal y como se estipula en el MCERL, la competencia comunicativa de la lengua está integrada por la combinación de las competencias lingüística, sociolingüística y pragmática e incluye la dimensión cultural en los componentes sociolingüístico -relativo al uso de la lenguay pragmático -que remite al uso funcional de la misma. Se hace ciertamente referencia al conocimiento del mundo y sociocultural cuya relevancia en el proceso de enseñanza-aprendizaje se reconoce habida cuenta de que no forma parte de la experiencia previa del aprendiz de lenguas quien lo ha de incorporar a su bagaje cultural. En consecuencia, el docente de LE ha de integrar el saber relacionado con la sociedad y la(s) cultura(s) de la(s) comunidade(s) en las que la lengua aprendida es hablada con el fin de que los aprendices tomen conciencia de la diversidad cultural y aprendan a identificar las similitudes y las diferencias.

Sin embargo, la situación actual revela ciertas carencias. En el Anejo B del MCERL, el proyecto de investigación del Consejo Nacional Suizo de Investigación Científica ${ }^{2}$ hace referencia a la dificultad de crear descriptores precisos que midan las competencias sociocultural y sociolingüística. Dicho proyecto tenía por objetivo desarrollar, analizar y validar las escalas de descriptores ilustrativos, que indican lo que el aprendiz 'puede hacer' cuando usa la lengua meta objeto de estudio. Sin embargo, con respecto a la evaluación de las competencias sociocultural y sociolingüística, los indicadores presentan ciertas deficiencias que parecen no garantizar la fiabilidad de los resultados en cuanto a la evaluación realizada. Ésta fue una de las razones que motivaron su eliminación. Estos problemas son puestos de manifiesto en el dicho anejo en los siguientes términos:

No está claro hasta qué punto este problema fue provocado: a) por el hecho de que éste sea un constructo separado del dominio de la lengua, b) por descriptores bastante imprecisos que se consideran problemáticos en los talleres de trabajo, o c) por las respuestas inconsistentes de los profesores que carecían del necesario conocimiento de sus alumnos (MECRL, 2002: 211).

En esta línea, García Mata y Barrios (2005: 265) subrayan: «a pesar de que asigna una enorme importancia a la adquisición de una competencia pluricultural, el marco de evaluación en forma de niveles que propone, se limita a valorar aspectos que constituyen la competencia lingüística». Por otro lado, el componente lingüístico aún parece ocupar un lugar privilegiado en la planificación del proceso educativo de las lenguas extranjeras en detrimento de lo sociocultural. Según Byram y Zarate (1997), buena parte de los docentes e incluso de los aprendices siguen concediendo prioridad a los aspectos formales del lenguaje en detrimento de los socioculturales y Gohard-Radenkovic (1999: 76) hace observar que en el aprendizaje de un idioma:

\footnotetext{
${ }^{2}$ En el Anejo B se presenta una visión general del proyecto del Consejo Nacional Suizo de Investigación Científica, que desarrolló los niveles comunes de referencia y sus descriptores ilustrativos (MECRL, 2001: 209-216).
} 
ce sont essentiellement les compétences linguistiques (compréhension orale, écrite, expression orale et écrite: les four skills) qui sont visées, afin que l'apprenant puisse atteindre une compétence minimale de communication dans une situation d'échanges langagiers.

Más recientemente en una investigación realizada por Corpas y Madrid (2009), quienes estudian el nivel de comprensión oral alcanzado por los estudiantes al finalizar la Educación secundaria obligatoria, entre otros resultados de gran interés, se observa que en tareas de tipo lingüístico tales como: "comprender de forma especifica textos orales" y "apreciar los usos imaginativos y creativos de la lengua inglesa", el porcentaje de alumnos y alumnas que consiguen estos objetivos es superior al $60 \%$, lo cual contrasta dramáticamente con tareas relacionadas con la dimensión sociocultural del tipo: "apreciar algunos significados sociales que transmite la lengua inglesa" y "apreciar algunos significados culturales transmitidos por la lengua inglesa", con porcentajes de éxito del 26,6\% y 38,3\%, respectivamente.

El interés por este estudio descriptivo que aquí se presenta obedece al deseo de analizar las percepciones del profesorado de lengua extranjera que reflejan convicciones, ideas, inclinaciones, etc. que abrigan en lo relativo a la importancia concedida a la integración del componente cultural en la enseñanza de la lengua extranjera. Pensamos que resulta de suma relevancia conocer la opinión del profesorado no sólo porque las creencias pueden determinar ciertas actitudes y actuaciones, sino porque de manera tácita pueden transmitirse valores al alumnado.

\section{Metodología}

\subsection{Estrategia de obtención de los datos: el cuestionario}

No habiendo encontrado en las fuentes estudiadas ni en la bibliografía consultada herramienta alguna que nos pareciera adecuada para esta investigación decidimos crear nuestro propio cuestionario. En una fase preliminar se desarrolló una prueba piloto que constituyó la base sobre la cual fundamentamos el diseño del cuestionario definitivo. La selección de ítems para este último se basó en un análisis factorial, el cual, si bien es una poderosa herramienta que permite decidir, dada una familia numerosa de ítems, cuáles de entre ellos presentan una cierta cohesión como grupo (Morgan, Leech, Gloeckner, Barrett, Jensen, y Quick, 2004) también tiene sus limitaciones.

En cuanto a la administración del mismo nos decantamos por la distribución personal a pesar del aspecto negativo que para el investigador conlleva ya que ha de asumir el coste en tiempo. Ese inconveniente quedó compensado por el alto índice de respuestas recibidas ya que sobre un total de 430 cuestionarios distribuidos, 370 fueron cumplimentados y entregados (86\%). La investigadora acudió a los centros educativos y explicó a uno de los docentes las razones que la impulsaban a suministrar dicho cuestionario, el propósito de la investigación, e insistió en garantizar el anonimato de los informantes. Al profesorado se le solicitaba en el cuestionario (ver tabla 1) que expresara su opinión sobre el contenido de los enunciados presentados a través de una escala de tipo Likert de 5 puntos ( $1=$ Muy en desacuerdo; $2=$ En desacuerdo; $3=\mathrm{Ni}$ de acuerdo ni en desacuerdo/Indeciso; 4= De acuerdo; $5=$ Muy de acuerdo). 
Tabla 1: Ítems presentados a los profesores y profesoras participantes en la encuesta, relativos a las necesidades y exigencias de la integración en la Unión Europea, la relevancia del componente lingüistico versus el componente cultural y los contenidos culturales que deberían enseñarse (versión en formato papel)

Indique su grado de acuerdo o desacuerdo con la formulación contenida en el ítem conforme a la escala siguiente:

1: Muy en desacuerdo; 2: En desacuerdo; 3: Ni de acuerdo ni en desacuerdo/Indeciso; 4: De acuerdo; 5: Muy de acuerdo.

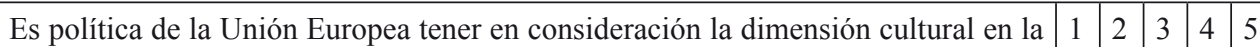
enseñanza de la LE.

\begin{tabular}{|l|l|l|l|l|l|}
\hline La enseñanza actual de la lengua extranjera cubre las necesidades de comunicación & 1 & 2 & 3 & 4 & 5
\end{tabular} intercultural en el marco de la Unión Europea.

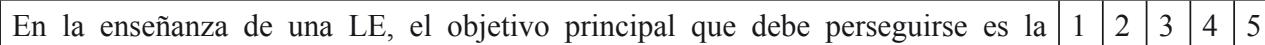
adquisición de una competencia lingüística.

\begin{tabular}{|lll|l|l|l|l} 
Mantener un equilibrio entre la enseñanza de la lengua y la cultura es & 1 & 2 & 3 & 4 & 5
\end{tabular} fundamental.

\begin{tabular}{|l|l|l|l|l|l|}
\hline Es preciso corregir los usos inadecuados de tipo sociocultural que hacen los alumnos & 1 & 2 & 3 & 4 & 5
\end{tabular} en la LE.

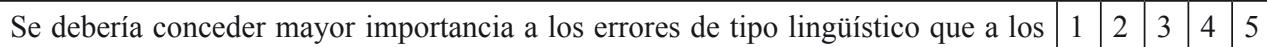
de tipo sociocultural.

\begin{tabular}{|lll|l|l|l} 
En el Proyecto Curricular de Centro se hace hincapié fundamentalmente en el & 1 & 2 & 3 & 4 & 5
\end{tabular} componente lingüístico.

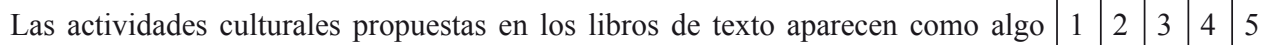
secundario frente al contenido lingüístico.

\begin{tabular}{|l|l|l|l|l|l|}
\hline Al seleccionar el libro de texto se debería tener en cuenta cómo aborda el componente & 1 & 2 & 3 & 4 & 5
\end{tabular} cultural.

Es preferible explicar en español los aspectos culturales.

\begin{tabular}{|l|l|l|l|l|l|}
\hline Los contenidos culturales que preferentemente deberían enseñarse son unos & 1 & 2 & 3 & 4 & 5
\end{tabular} conocimientos objetivos sobre realidades concretas (monumentos, eventos, hechos, personajes, etc.).

\begin{tabular}{|ll|l|l|l|l|l|}
\hline Los contenidos culturales que preferentemente deberían enseñarse son las & 1 & 2 & 3 & 4 & 5
\end{tabular} convenciones socioculturales verbales y no verbales.

\subsection{Población objeto de estudio}

La población estaba conformada por los profesores y profesoras que imparten docencia, de francés o inglés, en centros educativos de ESO y Bachillerato o en Escuelas Oficiales de Idiomas (en adelante, EEOOII), en las provincias de Málaga y Granada. Ante la imposibilidad de llegar a todo el colectivo, se distribuyó el cuestionario en el mayor número posible de centros educativos de ambas provincias. Éste fue cumplimentado por aquellos y aquellas profesores que accedieron voluntariamente a participar en la investigación. 


\subsection{Tipo de muestra y su representatividad}

Nuestro muestreo entraría en la categoría no aleatoria y, más específicamente, en la de muestra de conveniencia. Tras la recopilación de los cuestionarios y habida cuenta del tipo de muestra escogido, creímos preciso averiguar hasta qué punto los datos resultantes son aplicables al conjunto de la población y más concretamente, en nuestro caso, en relación a parámetros tales como: sexo, ubicación geográfica y lengua enseñada. Para ello, hicimos una comparación entre la distribución de nuestra muestra (ver tabla 2) y la del universo basándonos en los datos suministrados por la Consejería de Educación ${ }^{3}$. Al no proporcionar la Junta de Andalucía el número de profesores que imparten inglés o francés en las provincias de Granada y Málaga, se ha asignado a cada una de ellas un número de profesores teniendo en cuenta el peso relativo que, en el conjunto de la Educación secundaria, dicha provincia tiene.

Tabla 2: Comparación entre la muestra y la población objeto de estudio

\begin{tabular}{|l|c|c|c|c|}
\hline Porcentajes & \multicolumn{2}{|c|}{ Muestra } & \multicolumn{2}{c|}{ Población } \\
\hline Sexo & Mujer & Hombre & Mujer & Hombre \\
& $66,5 \%$ & $33,5 \%$ & $69 \%$ & $31 \%$ \\
\hline Idiomas & Inglés & Francés & Inglés $62 \%$ & Francés \\
& $58,9 \%$ & $41,1 \%$ & $38 \%$ \\
\hline Provincias & Málaga & Granada & Málaga & Granada \\
& $51,9 \%$ & $48,1 \%$ & $58 \%$ & $41 \%$ \\
\hline
\end{tabular}

En cuanto al tamaño de la muestra, la pregunta natural es ¿cuál es el número más pequeño de casos que nos permite extender los resultados a la población general con una probabilidad pequeña de error? Optamos por la muestra más amplia que nos podíamos permitir; un modo de comprobar que la muestra está saturada es dividirla aleatoriamente en dos mitades $A$ y $B$ y verificar que no hay diferencias estadísticamente significativas entre ambas. En efecto, si no existen diferencias entre el colectivo $A$ y el $B$ al agregar al colectivo $A$ sujetos del colectivo $B$, los resultados obtenidos en $A$ no variarán significativamente. En este caso, parece razonable concluir que si la muestra correspondiente al colectivo $A$ ya presenta saturación, tanto más la muestra completa (De la Torre Rodríguez Alberto, comunicación personal, septiembre de 2007) ${ }^{4}$.

\subsection{Estudio de la muestra}

La muestra utilizada, en nuestro trabajo, está formada por 370 participantes que está constituida por, prácticamente, el mismo número de profesores en Granada y en Málaga;

\footnotetext{
${ }^{3}$ Disponible en: http://www.juntadeandalucia.es/educacion/nav/contenido.jsp?pag=/Contenidos/Viceconsejeria/estadisticas_educativas, consultado en enero de 2007.

${ }^{4}$ De la Torre Rodríguez, Alberto (2007), Entrevista con Alberto de la Torre, licenciado en Matemáticas y en la especialidad de Estadística por la Universidad Complutense de Madrid.
} 
aproximadamente el doble de profesoras que de profesores y de cada diez docentes, seis son de inglés y cuatro de francés. Si bien la distribución de la muestra no coincide exactamente con la de la población, lo cual no es sorprendente habida cuenta del proceso seguido en la selección de la misma, las diferencias existentes están dentro de un margen razonable excepto quizá en la distribución por provincias.

\section{Procedimiento de análisis de datos}

Se procedió a un doble análisis de las respuestas dadas por los informantes a cada uno de los ítems. Para cada ítem del cuestionario se obtuvieron la distribución de frecuencias y su representación gráfica; la interpretación de los resultados se basaba en ambas. No incluimos las medias ya que la distribución de frecuencia relativa proporciona, en nuestra opinión, una información más completa e ilustrativa que la media dado que las variables toman solamente cinco valores distintos. Por otro lado, la media compensa entre los valores extremos por lo que no discrimina entre aquéllos casos en los que valores marcados se han concentrado a partes prácticamente iguales, en las opciones 1 y 5 , y aquéllas en las que la mayoría se ha inclinado, por ejemplo, por el 3. En cambio, en un gráfico con sólo 5 barras, como es nuestro caso, la diferencia es obvia. Por otra parte, en variables ordinales o nominales, la moda es un parámetro natural que se identifica a primera vista en un gráfico de barra o de sectores. Estudiamos, además, la posible existencia de diferencias estadísticamente significativas en las respuestas dadas por los encuestados en función de las siguientes variables: Sexo (Hombre y Mujer), Experiencia Docente (entre 1 y 10 años, entre 11 y 20 años y más de 20 años), Nivel de enseñanza (ESO, ESO y Bachiller; EEOOII), Idiomas (Francés e Inglés) y Provincias (Granada y Málaga). Se utilizaron las pruebas de Mann-Whitney o Kruskal-Wallis en función del número de niveles de la variable independiente (Morgan et al., 2004: 50). Para estudiar posibles correlaciones entre los ítems usamos la Tau-b de Kendall ya que la escala de nuestro estudio es ordinal.

\section{Resultados}

\subsection{Resultados en relación a los ítems sobre la importancia que la Unión Europea otorga a la dimensión cultural}

4.1.1. Item 1. «Es política de la Unión Europea tener en consideración la dimensión cultural en la enseñanza de la LE»

Un 59\% de los profesores de LE optaron por los valores máximos 4 y 5 y, sólo un $12 \%$, por los valores 1 y 2 , con un significativo porcentaje de indecisos (29\%); tan sólo un $2 \%$ de los encuestados se inclinaron por la opción muy en desacuerdo. Existía, pues, un amplio consenso entre el profesorado sobre la importancia que la Unión Europea otorga a la dimensión cultural en el campo de la enseñanza de una lengua extranjera.

Se realizó un análisis del cruce del ítem 1 con cada una de las variables descriptivas no encontrando diferencias significativas en ningunos de ellos (Sexo: $p=0,108$; Nivel de 
enseñanza: $p=0,638$, Experiencia docente: $p=0,917$; Idiomas: $p=0,103$; Provincias: $p=0,142$ ). Sin embargo, cabe destacar, que el profesorado de francés consideraba en mayor grado que el de inglés que, en la política de la Unión europea, es una prioridad integrar el componente cultural en la enseñanza de las lenguas. En efecto, el porcentaje de los docentes que estaba de acuerdo o muy de acuerdo es de un 56,6\% entre los que impartían inglés y de un $63,8 \%$ entre los de francés. Por otro lado, la moda entre los profesores de francés es muy de acuerdo, con un $34 \%$, mientras que, entre los profesores de inglés, hay dos modas: de acuerdo e indeciso, ambas con un $32,4 \%$.

4.1.2. Ítem 2. «La enseñanza actual de la lengua extranjera cubre las necesidades de comunicación intercultural en el marco de la Unión Europea»

El rasgo más sobresaliente de los resultados es la gran diferencia -al menos diez puntos porcentuales- entre la moda (en desacuerdo) y cualquiera de los demás valores. Si agrupamos las opciones en desacuerdo y muy en desacuerdo obtenemos que un significativo 58,3\% del profesorado encuestado estimaba que la enseñanza de la LE no satisfacía las necesidades de comunicación intercultural en el marco de la Unión Europea. Solamente un 14,3\% opinaba de un modo positivo; pero de este porcentaje tan sólo el 3,2\% estaba totalmente convencido. Por último, el porcentaje $(27,3 \%)$ de los profesores que no estaban ni de acuerdo ni en desacuerdo es similar al del ítem anterior.

Es interesante observar que el porcentaje de quienes estaban de acuerdo o muy de acuerdo en que la Unión Europea concedía importancia a los aspectos culturales es prácticamente el mismo que el de quienes creían que el estado de la integración de la cultura no era satisfactorio. El análisis bivariable no detectó diferencias estadísticamente significativas en función de las variables descriptivas (Sexo: $p=0,217$; Nivel de enseñanza: $p=0,387$; Experiencia docente: $\mathrm{p}=0,781$; Idiomas: $\mathrm{p}=0,120$; Provincias: $\mathrm{p}=0,124$ ).

\subsection{Resultados en relación a los ítems sobre la importancia relativa del componente lingüístico frente al componente cultural}

4.2.1. Ítem 3. "En la enseñanza de una LE, el objetivo principal que debe perseguirse es la adquisición de una competencia lingüística»

De los datos obtenidos se deduce que, abrumadoramente, el profesorado creía que en la enseñanza de una LE no era suficiente impartir unos contenidos estrictamente lingüísticos. Concretamente, un $63 \%$ de los profesores estaba en desacuerdo o muy en desacuerdo con lo enunciado en el ítem; dicho de otro modo, opinaba que una sólida base en los niveles fonológico, morfosintáctico y léxico no proporcionaba los conocimientos suficientes para alcanzar una comunicación eficaz, frente a un $20,4 \%$ que estimaba que bastaría con la competencia lingüística.

En lo relativo al análisis bivariable, en este ítem hallamos diferencias significativas en función de las variables Sexo $(\mathrm{p}=0,029)$, Idiomas $(\mathrm{p}=0,042)$, Nivel de enseñanza $(\mathrm{p}=0,000)$ y Provincias $(\mathrm{p}=0,002)$. La variable descriptiva que presenta mayor influencia es la de Sexo. En efecto, advertimos que las mujeres $(66,7 \%)$ eran más reacias que los hombres $(55,7 \%)$ a afirmar que el objetivo principal de la enseñanza de la LE debiera ser el componente 
lingüístico. Observamos también que los profesores de francés no estaban de acuerdo con la afirmación del ítem en un $69,6 \%$ frente a un $58,9 \%$, de los de inglés. Llama la atención que aquéllos que impartían docencia en las EEOOII, estaban muy en desacuerdo (48,3\%) en un porcentaje que casi duplicaba a los profesores de ESO (26\%) y ESO y Bachiller $(27,7 \%)$. Además, el profesorado informante de EEOOII manifestaba estar muy de acuerdo en menos de un $2 \%$ frente a un $12,1 \%$ en el caso del profesorado de ESO y un 7,6\% en el de ESO y Bachiller. Resulta lícito deducir que el profesorado de las EEOOII concedía también importancia a los demás aspectos (y considera que debe complementarse la enseñanza de la LE con las dimensiones sociolingüística, sociocultural y pragmática). Los profesores y las profesoras participantes en la encuesta de la provincia de Granada estaban más en desacuerdo $(72,1 \%)$ que sus compañeros de Málaga $(54,7 \%)$ con que las demás dimensiones fueran menos valoradas en el proceso de enseñanza de la LE.

\subsection{2. Ítem 4. «Mantener un equilibrio entre la enseñanza de la lengua y la cultura} es fundamental»

Si comparamos los resultados de este ítem con los del anterior, nos percatamos de que los y las docentes, que no estaban de acuerdo en que el objetivo último de la enseñanza de una lengua extranjera fuera el dominio del código lingüístico, reflejaban, por término medio, a través de las opciones marcadas, que debería mantenerse un equilibrio entre los aspectos culturales y formales. A menor valoración de la afirmación del ítem 3 corresponde una mayor valoración de la afirmación del ítem 4. Es más, el coeficiente de correlación no paramétrico de Kendall viene a confirmar esta apreciación al dar un valor negativo $(-, 114)$.

El estudio de la tabla de contingencia nos llevó a concluir que el profesorado de francés estaba más de acuerdo con establecer un equilibrio entre el componente lingüístico y el cultural que el profesorado de inglés, siendo esta diferencia estadísticamente significativa $(p=0,019)$. Sin embargo, en el análisis bivariable no se obtuvieron diferencias estadísticamente significativas en función de las variables descriptivas (Sexo: $p=0,256$; Nivel de enseñanza: $\mathrm{p}=0,611$; Experiencia docente: $\mathrm{p}=0,450$; Provincias: $\mathrm{p}=865$ ).

4.2.3. Ítem 5. "Es preciso corregir los usos inadecuados de tipo sociocultural que hacen los alumnos en la LE»

En este ítem, la mayoría de los profesores y de las profesoras se inclinaba por las opciones 4 o 5 en un porcentaje abrumador; casi ocho de cada diez estimaban que es necesario que el alumnado sea consciente de la relevancia de ese tipo de error para un buen entendimiento con los hablantes de la lengua objeto de estudio. El coeficiente de correlación entre los ítems 4 y 5 , cuyos enunciados hacen hincapié en la necesidad de incluir los aspectos socioculturales, es positivo $(0,250)$.

Al igual que en el ítem anterior, según se desprende de los datos, el profesorado de francés se inclina, de forma más acentuada, por la corrección de los usos inadecuados de tipo sociocultural $(p=0,002)$. Sin embargo, en el análisis bivariable de las demás variables descriptivas no se hallaron diferencias estadísticamente significativas (Sexo: $p=0,899$; Nivel de enseñanza: $p=0,744$; Experiencia docente: $p=0,332$; Provincias: $p=596$ ). 
4.2.4. Ítem 6. «Se debería conceder mayor importancia a los errores de tipo lingüístico que a los de tipo sociocultural»

Pese a que una gran mayoría (77\%) del profesorado afirmaba de un modo categórico la necesidad de proceder a la corrección de los usos inadecuados de tipo sociocultural, un $62,2 \%$ contestaba que aún más importante era corregir los errores de tipo lingüístico. Por tanto, esos resultados parecen sugerir que si bien los profesores estaban persuadidos de la necesidad de tener en consideración los usos inadecuados de tipo sociocultural, seguían atribuyendo mayor relevancia al contenido lingüístico, o dicho de otro modo, primaban la construcción correcta desde un punto de vista gramatical.

Realizamos las pruebas no paramétricas correspondientes y obtuvimos una correlación positiva y estadísticamente significativa con el ítem 3 . Constatamos que existía una correlación positiva entre los ítems 3 y $6(0,128)$, los cuales expresan un predominio de las formas de la lengua sobre los aspectos sociolingüísticos y socioculturales.

En el análisis bivariable, la única diferencia estadísticamente significativa se da en relación a la variable Idiomas $(\mathrm{p}=0,026)$, siendo los profesores de inglés los que concedían mayor importancia a los errores de tipo lingüístico con respecto a los de tipo sociocultural. En nuestra opinión, estos datos confirman lo apuntado en el ítem 4 en el que detectamos que éstos últimos estaban menos de acuerdo que el profesorado de francés en que debía existir un equilibrio entre las dimensiones lingüísticas y culturales.

En el análisis bivariable de las demás variables descriptivas no se encontraron diferencias estadísticamente significativas (Sexo: $p=0,400$; Nivel de enseñanza: $p=0,304$; Experiencia docente: $\mathrm{p}=0,180$; Provincias: $\mathrm{p}=0,834$ ).

\subsection{5. Ítem 7. «En el Proyecto Curricular de Centro se hace hincapié fundamentalmente} en el componente lingüístico»

Casi la mitad (cuatro de cada diez profesores) reconocía que el Proyecto Curricular de su centro se focalizaba sustancialmente en las formas lingüísticas. Si tenemos en cuenta que un 13,4\% compartía esta opinión, incluso en mayor grado (muy de acuerdo), podríamos llegar a la conclusión de que, en el Proyecto Curricular de Centro, no se otorgaba a la dimensión cultural la importancia que, según el profesorado, debería concedérsele habida cuenta de que en el ítem 4, un $63,24 \%$ de los encuestados marcaba la opción de acuerdo o muy de acuerdo y en el ítem 5, ese porcentaje alcanzaba hasta el $77 \%$.

El colectivo de los hombres estimaba en mayor grado que sus compañeras que, en el Proyecto de Curricular de su centro, se primaba el componente lingüístico en relación al cultural $(\mathrm{p}=0,047)$. En el análisis bivariable de las variables descriptivas restantes no se obtuvieron diferencias estadísticamente significativas (Nivel de enseñanza: $p=0,943$; Experiencia docente: $\mathrm{p}=0,479$; Idiomas: $\mathrm{p}=0,832$; Provincias: $\mathrm{p}=0,460$ ).

4.2.6. Item 8. «Las actividades culturales propuestas en los libros de texto aparecen como algo secundario frente al contenido lingüístico»

Con los ítems 8 y 9 buscábamos información sobre la relevancia que se otorgaba al conocimiento cultural en los libros de texto existentes en el mercado editorial, según el 
profesorado de LE que había cumplimentado el cuestionario. Un 64,33\% estaban de acuerdo o muy de acuerdo en que el componente cultural no gozaba de un lugar preferente en relación al componente lingüístico. De ello, podríamos derivar que existe en los libros de texto carencias apreciables en cuanto al tratamiento específico de la dimensión cultural. Sin embargo, en el ítem 4 un $64,24 \%$ se declaraba a favor de una planificación en la que se dispensara a los contenidos culturales un tratamiento adecuado. No encontramos ninguna diferencia estadísticamente significativa con respecto a las variables descriptivas (Sexo: $\mathrm{p}=$ 0,929; Nivel de enseñanza: $p=0,218$; Experiencia docente: $p=0,415$; Idiomas: $p=0,504$; Provincias: $\mathrm{p}=0,167)$.

\subsection{7. Ítem 9. "Al seleccionar el libro de texto se debería tener en cuenta cómo aborda} el componente cultural»

Según se desprende de las respuestas dadas a este ítem, una gran mayoría (siete de cada diez profesores) estaba de acuerdo en que un criterio a primar, cuando se procede a la selección del libro de texto que va a ser utilizado en el aula de idioma, es el modo en que se plantea y se presenta el contenido cultural. Al igual que en el ítem anterior no hallamos ninguna diferencia estadísticamente significativa en relación a las variables descriptivas planteadas por el estudio (Sexo: $p=0,234$; Nivel de enseñanza: $p=0,092$; Experiencia docente: $\mathrm{p}=0,450$; Idiomas: $\mathrm{p}=0,295$; Provincias: $\mathrm{p}=0,226$ ).

\subsection{8. Ítem 10. «Es preferible explicar en español los aspectos culturales»}

Decidimos incluir este ítem porque parece razonable esperar que si el profesorado concede suficiente importancia al contenido que alude propiamente a la cultura, no dudará en explicarlo en la lengua castellana para asegurarse de que el alumnado entienda y capte las sutilezas de los elementos culturales. En efecto, es muy probable que estos matices no puedan ser apreciados en su justa medida, si solamente se expusieran en la lengua que el alumnado aún no domina. Si bien un 38\% se muestra indeciso, algo más de cuatro de cada diez profesores $(44,5 \%)$ se inclina por presentarlos en la lengua materna del alumno, y menos de un $18 \%$ discrepa.

Lo llamativo del ítem 10 estriba en que existían diferencias sustanciales entre los profesores y las profesoras de EEOOII y los demás docentes (ver figura 1). El profesorado de EEOOII optaba abrumadoramente $(43,4 \%)$ por hacer uso de la lengua extranjera para exponer los aspectos culturales al alumnado, frente al $8 \%$ de los profesores de ESO y el $14 \%$ de los de ESO y Bachillerato que se decantaba por recurrir a la lengua materna. Podríamos hallar una posible explicación para este hecho examinando con más detenimiento el perfil del alumnado que acude a esos centros. 


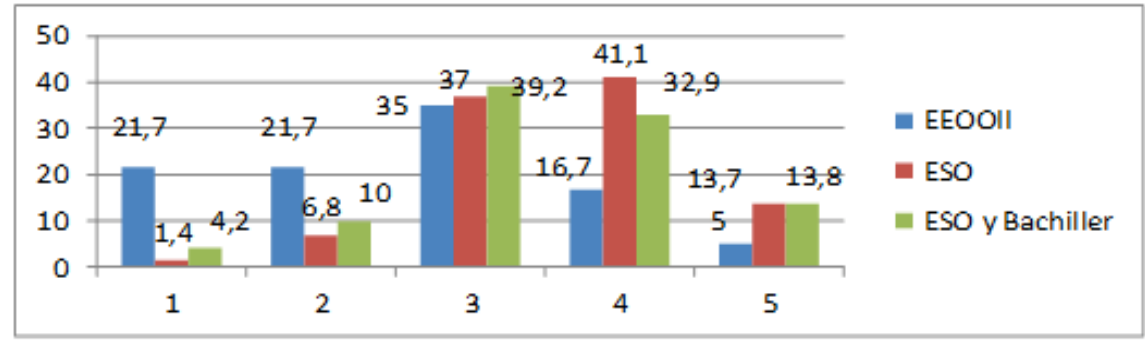

Figura 1: Cruce entre el item 10 «Es preferible explicar en español los aspectos culturales» y la variable descriptiva Nivel de Enseñanza

La prueba de Kruskal Wallis confirma que las conclusiones obtenidas de la observación del gráfico corresponden a diferencias significativas entre los tres grupos. En este ítem hallamos diferencias significativas en la variable Nivel de enseñanza $(p=0,000$. En el análisis bivariable de las demás variables descriptivas no se hallaron diferencias estadísticamente significativas (Sexo: $\mathrm{p}=0,055$; Experiencia docente: $\mathrm{p}=0,894$; Idiomas: $\mathrm{p}=0,562$; Provincias: $\mathrm{p}=0,312)$.

\subsection{Resultados en relación a los ítems sobre los contenidos que deberían enseñarse}

4.3.1. Ítem 11. «Los contenidos culturales que preferentemente deberían enseñarse son unos conocimientos objetivos sobre realidades concretas (monumentos, eventos, hechos, personajes, etc.)»

Los profesores se mostraban mayoritariamente a favor de enseñar unos conocimientos concretos sobre cultura ya que el $61 \%$ optó por las opciones de acuerdo o muy de acuerdo y sólo un $13 \%$ de los encuestados marcó las opciones en desacuerdo o muy en desacuerdo. No se apreciaron, en este ítem, diferencias significativas en función de las variables descriptivas (Sexo: $p=0,321$; Nivel de enseñanza: $p=0,827$; Experiencia docente: $p=0,308$; Idiomas: $\mathrm{p}=0,321$; Provincias: $\mathrm{p}=0,250$ ).

4.3.2. Ítem 12. «Los contenidos culturales que preferentemente deberían enseñarse son las convenciones socioculturales verbales y no verbales»

En relación con el ítem anterior, el porcentaje de los profesores que estaba de acuerdo o muy de acuerdo disminuye sensiblemente, concretamente en un $25 \%$. Es razonable pensar que quienes pensaban que preferentemente debería enseñarse realidades concretas no estarían de acuerdo en que lo más importante fuesen las convenciones socioculturales. Era pues de esperar una correlación negativa entre ambos ítems; sorprendentemente aunque pequeño el coeficiente de correlación es positivo (Tau de Kendall 0,052). Esto parece indicar que no se interpretó en un sentido excluyente el adverbio 'preferentemente'.

Si realizamos un análisis más detallado de las tablas de contingencia, comprobamos que en las distribuciones, correspondientes al nivel de enseñanza EEOOII, no se advierten 
prácticamente diferencias en las respuestas entre los ítems 11 y 12 , con un $61 \%$ y 49,1\% respectivamente sumando las opciones de acuerdo y muy de acuerdo. Cabe pues plantearse que la enseñanza de ambos aspectos era relevante para los informantes que contestaron a los ítems, aunque no obstante parecían poner más énfasis en las realidades concretas. En contraposición, las distribuciones correspondientes al nivel de enseñanza de ESO indican una tendencia contraria, pues las opciones de acuerdo y muy de acuerdo en el ítem 11 suman un $64,4 \%$ y en el ítem 12 tan sólo un 37,5\% y en lo que concierne a los docentes de ESO y Bachiller (61,4\% y 48,3\%, respectivamente) (ver tablas 3 y 4$)$.

\section{Tabla 3. Ítem 11 y variable Nivel de enseñanza}

\begin{tabular}{|c|c|c|c|c|c|}
\hline & \multicolumn{3}{|c|}{ Niveles de enseñanza } & \multirow[b]{2}{*}{ Total } \\
\hline & & ESO & $\begin{array}{c}\text { ESO y } \\
\text { Bachiller }\end{array}$ & EEOOII & \\
\hline \multirow{5}{*}{$\begin{array}{l}\text { Los contenidos culturales que preferentemente } \\
\text { deben enseñarse son unos conocimientos objetivos } \\
\text { sobre realidades concretas (monumentos, eventos, } \\
\text { hechos, personajes, etc.). }\end{array}$} & 1 & $4,1 \%$ & $2,1 \%$ & $1,8 \%$ & $2,5 \%$ \\
\hline & 2 & $5,5 \%$ & $11,9 \%$ & $14,0 \%$ & $10,9 \%$ \\
\hline & 3 & $26,0 \%$ & $24,6 \%$ & $28,1 \%$ & $25,4 \%$ \\
\hline & 4 & $46,6 \%$ & $39,4 \%$ & $35,1 \%$ & $40,2 \%$ \\
\hline & 5 & $17,8 \%$ & $22,0 \%$ & $21,1 \%$ & $21,0 \%$ \\
\hline Total & & $100,0 \%$ & $100,0 \%$ & $100,0 \%$ & $100,0 \%$ \\
\hline
\end{tabular}

Tabla 4. Ítem 12 y variable Nivel de enseñanza

\begin{tabular}{|c|c|c|c|c|c|}
\hline & \multicolumn{3}{|c|}{ Niveles de enseñanza } & \multirow[b]{2}{*}{ Total } \\
\hline & & ESO & $\begin{array}{c}\text { ESO y } \\
\text { Bachiller }\end{array}$ & EEOOII & \\
\hline \multirow{5}{*}{$\begin{array}{l}\text { Los contenidos culturales que preferentemente } \\
\text { deben enseñarse son las convenciones } \\
\text { socioculturales verbales y no verbales. }\end{array}$} & 1 & $8,3 \%$ & $2,9 \%$ & $1,7 \%$ & $3,8 \%$ \\
\hline & 2 & $20,8 \%$ & $17,6 \%$ & $13,6 \%$ & $17,6 \%$ \\
\hline & 3 & $33,3 \%$ & $31,1 \%$ & $35,6 \%$ & $32,2 \%$ \\
\hline & 4 & $31,9 \%$ & $32,8 \%$ & $27,1 \%$ & $31,7 \%$ \\
\hline & 5 & $5,6 \%$ & $15,5 \%$ & $22,0 \%$ & $14,6 \%$ \\
\hline Total & & $100,0 \%$ & $100,0 \%$ & $100,0 \%$ & $100,0 \%$ \\
\hline
\end{tabular}

Además, así como en el ítem 11 no había influencia de las variables descriptivas, en relación a este ítem se dan diferencias significativas, por un lado, entre los distintos niveles de enseñanza, mostrando un mayor grado de acuerdo los docentes de las EEOOII $(p=0,032)$ $\mathrm{y}$, por el otro, los de francés $(\mathrm{p}=0,004)$. En cuanto al análisis bivariable de las demás variables descriptivas no se hallaron diferencias estadísticamente significativas (Sexo: $p=0,612$; Experiencia docente: $\mathrm{p}=0,171$; Provincias: $\mathrm{p}=0,499)$. 


\section{Conclusiones}

De los resultados obtenidos en relación al primer grupo de ítems puede inferirse que si bien los participantes en la encuesta eran mayoritariamente de la opinión de que para la Unión Europea es de capital interés que el alumnado consiga, además de una sólida competencia lingüística, una adecuada competencia cultural que le permita actuar y comunicarse con eficiencia y éxito en los eventos sociales, no es menos cierto que estimaban que la situación distaba mucho de proveer dichas necesidades y era, por tanto, manifiestamente mejorable. En lo relativo al resto de los ítems, podemos concluir de las respuestas dadas que el profesorado opina que debería existir un equilibrio entre la enseñanza de los exponentes lingüísticos y los elementos culturales. Es decir, el profesorado encuestado sostiene que si bien el tratamiento del componente lingüístico es una condición necesaria para dominar un idioma, ciertamente no es suficiente para un uso eficiente del mismo. Esto concuerda con la afirmación expresada por Porcher (1988: 92) al afirmar que el aprendizaje de una lengua precisa

percevoir les systèmes de classement à l'aide desquels fonctionne une communauté sociale et, par conséquent, d'anticiper, dans une situation donnée, ce qui va se passer (c'est-à-dire quels comportements il convient d'avoir pour entretenir une relation adéquate avec les protagonistes de la situation).

La comunicación entendida como una práctica social no puede limitarse a la comunicación lingüística ya que los aspectos extralingüísticos entran en juego en el acto comunicativo y su ignorancia puede ser causa de malentendidos. Los resultados de los ítems 3, 4 y 5 apuntan en ese sentido. Aun así, a la luz del análisis de las respuestas dadas por los encuestados al ítem 6 se aprecia que los y las docentes parecen adjudicar una mayor relevancia a las formas lingüísticas y a la corrección de los errores gramaticales, debido, quizá, al énfasis que pone en estos dos últimos aspectos el Proyecto Curricular del centro en el que imparten docencia. En cuanto al material editorial, una amplia mayoría, al seleccionar el libro de texto, se inclinaba por escoger aquél que trataba adecuadamente el componente cultural a pesar de las carencias que, en su opinión, el mercado editorial presentaba a este respecto. Por último, se hallan diferencias entre los tres colectivos (ESO, ESO y Bachiller y EEOOII) en lo que respecta a los contenidos que deberían enseñarse. Si bien todos optaban por enseñar conocimientos objetivos sobre realidades concretas, hay diferencias de grado siendo los profesores de ESO los más partidarios de primar esos elementos y los de EEOOII, los que menos.

\section{Bibliografía}

Bourguignon, C. (2007). "Apprendre et enseigner les langues dans la perspective actionnelle: le scénario d'apprentissage-action", disponible en: http://www.aplv-languesmodernes.org/spip. php?article865, consultado en marzo, 2008.

Conseil de 1'Europe (2001). Cadre européen commun de référence pour les langues. Apprendre, enseigner, évaluer. Paris: Les Éditions Didier. 
Corpas, M. D. y Madrid, D. (2009). "Desarrollo de la comprensión oral en Inglés como LE al término de la Educación Secundaria Obligatoria española", en Porta Linguarum, 11:129145.

García, J. y Barrios, M.E. (2005). "El tratamiento de la cultura en el Marco común europeo de referencia para las lenguas", en M.R. Luengo González, G. de la Maya Retamar y P. Gutiérrez Estéban (eds.), Actas del VIII Simposio Internacional de la Sociedad Española de Didáctica de la Lengua y la Literatura. Cultura, interculturalidad y Didáctica de la lengua y la literatura. Badajoz: Diputación de Badajoz, 261-266.

Girard, D. (1981). Linguistique appliquée et didactique des langues. Paris: Armand Colin.

Gohard-Radenkovic, A. (1999). Communiquer en langue étrangère. Des compétences culturelles vers des compétences linguistiques. Bern: Peter Lang.

Julié K. y Perrot, L. (2008). Enseigner l'anglais. Paris: Hachette Education.

Consejo de Europa (2002). Marco común europeo de referencia para las lenguas: aprendizaje, enseñanza, evaluación. Madrid: Ministerio de Educación, Cultura y Deporte.

Morgan, G., Leech, N., Gloeckner, G., Barrett, K., Jensen, L. y Quick D. (2004). SPSS for Introductory Statistics: Use and Interpretation. Mahwah, NJ: Lawrence Erlbaum Associates.

Porcher, L. (1988). "Programmes, progrès, progressions, projets dans l'enseignement/apprentissage d'une culture étrangère", en Etudes de linguistique appliquée, 69: 90-100.

Puren, Ch. (2009). "La problématique de la compétence culturelle dans le cadre de la mise en oeuvre de la nouvelle perspective actionnelle", disponible en: http://www.aplv-languesmodernes.org/spip.php?article2673, consultado en marzo, 2010.

Saville-Troike, M. (1982). The ethnography of communication. An introduction. Oxford: Basil Blackwell Publisher. 